\title{
Macroprogramming in the Internet of Things: A Systematic Mapping Study
}

\author{
Thalia S. Santana ${ }^{1,2}$, Iwens G. Sene Júnior ${ }^{1}$, Renato F. Bulcão-Neto ${ }^{1}$ \\ ${ }^{1}$ Instituto de Informática - Universidade Federal de Goiás (UFG) \\ Caixa Postal 131 - 74.690-900 - Goiânia - GO - Brasil \\ ${ }^{2}$ Instituto Federal Goiano - Campus Ceres (IF Goiano) \\ Caixa Postal 51 - 76.300-000 - Ceres - GO - Brasil \\ thaliasantana@discente.ufg.br, \{iwens, rbulcao\}@ufg.br
}

\begin{abstract}
The macroprogramming concept relates to the ability to abstract lowlevel details from a variety of devices. In this sense, programming solutions for the Internet of Things (IoT) with macroprogramming can be an alternative to the challenges of volume and heterogeneity. This paper describes a systematic mapping on macroprogramming in IoT and wireless sensor networks from 2004 to 2020. As a result, we verify the recurrence of abstractions in the network infrastructure, highlighting the use of frameworks in one-third of the applications, contributing to provide an overview of the use of macroprogramming by researchers in different areas of knowledge.
\end{abstract}

Resumo. O conceito de macroprogramação relaciona-se à capacidade de abstrair detalhes de baixo nível de variados dispositivos. Neste sentido, programar soluções para Internet das Coisas (IoT) com a macroprogramação pode ser uma alternativa perante aos desafios de volume e heterogeneidade. Este trabalho realizou um mapeamento sistemático da literatura quanto a macroprogramação no âmbito de IoT e redes de sensores sem fio de 2004 a 2020. Entre os resultados obtidos, foi possível verificar a recorrência de abstrações na infraestrutura de rede, destacando-se o emprego de frameworks em um terço das aplicações, contribuindo para propiciar uma visão geral do emprego de macroprogramação pelos pesquisadores em distintas áreas de conhecimento.

\section{Introdução}

A Internet das Coisas (IoT - Internet of Things) tem transformado a forma de interação entre dispositivos, pessoas e coisas. Este conceito relaciona-se com um paradigma tecnológico capaz de prover comunicação entre sensores, atuadores e processadores, presentes em objetos conectados em prol de um mesmo intuito significativo [Sethi and Sarangi 2017]. Combinada às redes de sensores sem fio (WSN, Wireless Sensor Networks), IoT compõe um domínio diversificado com potencialidade em aplicações variadas [Sugihara and Gupta 2008], como educação, saúde e meio ambiente.

Envolvendo hardware, software e outros recursos computacionais, há complexidade em propor aplicações distribuídas dada a dificuldade dos detalhes de baixo nível dos dispositivos [Lepekhin et al. 2019]. Outro ponto é a heterogeneidade de componentes e linguagens existentes, o que torna esta tarefa difícil para programadores 
[Choochaisri et al. 2012]. Soma-se a isso a atuação em contextos altamente dinâmicos [Awan et al. 2007], por exemplo, de sensores de tráfego urbano com detecção em tempo real ou sensores de avaliação de fatores de qualidade do solo em lavouras.

Para contribuir com o processo de desenvolvimento de tecnologias no ambiente das WSN e IoT, uma das formas de dirimir este problema é a utilização da macroprogramação (ou macroprogramming). O principal intuito é simplificar a construção de sistemas, utilizando mecanismos de abstrações na rede, comportando-se como um único conjunto distribuído [Choochaisri et al. 2012], o que possibilita uma programação mais simplificada aos desenvolvedores de aplicações, que não necessitarão conhecer os detalhes específicos de cada componente distinto.

A macroprogramação refere-se à programação de alto nível, produzindo abstrações onde os componentes são vistos no comportamento global, gerando programas mais concisos e robustos [Newton and Welsh 2004]. Assim, os diversos nós presentes em uma aplicação podem ser entendidos como uma coleção de objetos, que devido à necessidade de possíveis alterações, não precisam mais ser modificados e programados um a um. As alterações serão efetuadas uma única vez no macroprograma, o qual é capaz de abstrair e adaptar como um todo os nós interligados em tempo de compilação ou de execução, o qual ainda é um grande desafio [Hussein et al. 2017].

Este artigo investiga como os trabalhos vêm aplicando a macroprogramação em sistemas inerentes à IoT e WSN, e para isso, foi efetuado um mapeamento sistemático da literatura (MSL) [Petersen et al. 2015]. As contribuições deste estudo colaboram para o estado da arte do paradigma de macroprogramação, ao identificar o nível das adaptações realizadas, com tendências em abstrações aplicadas ao grupo de nós. Também é possível compreender de que maneira esse tipo de solução tem sido empregada em WSN e seu consequente crescimento em IoT nos últimos anos.

O artigo está assim organizado: a Seção 2 apresenta estudos relacionados; a Seção 3 detalha o protocolo de mapeamento sistemático até à extração de dados dos artigos; a Seção 4 responde às questões de pesquisa deste MSL ao discutir os resultados obtidos; e a Seção 5 descreve as considerações finais e trabalhos futuros.

\section{Trabalhos Relacionados}

Esta seção descreve estudos sistemáticos da literatura que versam sobre o tema macroprogramação em IoT e WSN.

No estudo de [Mottola and Picco 2011], fez-se um levantamento de estado da arte de programação para WSN. Foi realizada a identificação e classificação das abordagens existentes, verificando suas principais diferenças e identificando seus contextos de aplicação. Os autores propuseram como contribuição uma taxonomia às aplicações de WSN, na qual os artigos analisados foram mapeados.

Um levantamento do tipo survey sobre sistemas auto-adaptativos foi realizado em [Krupitzer et al. 2015], no qual descreve-se uma taxonomia para descrição das dimensões de tempo, razão, técnica, nível e adaptação ao controle. Já em [Lepekhin et al. 2019], foram identificados os principais desafios relativos a soluções de IoT por meio de um mapeamento sistemático. Foram encontradas seis categorias de desafios, os quais envolvem oportunidades em diferentes cenários de aplicação e tecnologias de comunicação. 
Sendo assim, esta pesquisa segue características de estudos anteriores ao abranger contextos de WSN e IoT, contudo, expande-se ao avaliar o paradigma sob a perspectiva de adaptação nesses contextos. Os estudos relacionados concentraram-se em verificar estes temas de forma isolada, o presente trabalho diferencia-se ao prover um mapeamento sistemático considerando aplicações de macroprogramação com adaptação na infraestrutura, como forma de compreender as abstrações realizadas e suas propostas em um recorte temporal nos últimos 16 anos.

\section{Métodos}

Este trabalho empregou o método de mapeamento sistemático da literatura (MSL), que visa a categorização e classificação dos resultados obtidos em prol de uma visão geral de uma área de pesquisa [Petersen et al. 2015]. Como abordagem passível de reprodutibilidade, um MSL inclui três etapas: planejamento, condução e publicação de resultados.

$\mathrm{Na}$ etapa de planejamento do MSL, deve-se definir o objetivo, as questões de pesquisa, a estratégia de busca, a string de busca, os critérios de seleção de estudos e um formulário para a extração de dados de cada estudo considerado relevante. Na etapa de condução do MSL, deve-se executar a estratégia de busca com a string definida, aplicar os critérios de seleção ao ler os metadados de cada estudo encontrado, ler na íntegra cada estudo selecionado e preencher o formulário definido e analisar e sintetizar os dados extraídos para responder às questões de pesquisa. Por fim, a etapa de publicação dos resultados, dá-se na forma de redação de relatórios e/ou artigos científicos.

O planejamento e a condução do MSL foram realizados com o apoio da ferramenta Parsif.al ${ }^{1}$, enquanto que a extração, a análise e a síntese dos dados extraídos dos artigos relevantes deu-se por meio de planilhas do Google Sheets. A documentação do MSL deu-se com o apoio de ambas as ferramentas, incluindo a geração de gráficos. Informações adicionais sobre este MSL, incluindo o protocolo e detalhes dos resultados estão disponíveis ${ }^{2}$.

Seguindo as etapas do MSL, o objetivo deste MSL é mapear os estudos primários que adotam macroprogramação e adaptação nas áreas de WSN e IoT. Para isso, foram definidas cinco questões de pesquisa $(\mathrm{QP})$, a saber:

- QP1: Quanto à macroprogramação, quais áreas de aplicação são enfocadas?

- QP2: Quando e em quais veículos os estudos primários têm sido publicados?

- QP3: Em qual(is) nível(is) ocorrem a adaptação e qual(is) são os tipos de abstrações na infraestrutura?

- QP4: Como adaptações são realizadas em pesquisas aplicadas a WSN e IoT?

- QP5: Quais são os problemas apontados no contexto da adaptabilidade nos domínios de aplicação estudados?

A estratégia de busca selecionada inclui o uso de mecanismos de busca das bibliotecas digitais ACM Digital Library, IEEE Xplore e Scopus, dada sua relevância e abrangência para a Ciência da Computação. O passo seguinte foi a definição da string de busca juntamente com um especialista em macroprogramação e Internet das Coisas. Uma sequência de testes-piloto foi realizada para o refinamento da string de busca até haver um balanceamento entre a precisão e a recuperação nos resultados das buscas-piloto. A seguir, apresenta-se a string de busca final:

\footnotetext{
${ }^{1}$ Disponível para acesso em: http://parsif .al/

${ }^{2}$ Disponível para acesso em: http: / / bit. ly / 3t HUOD l
} 
(macroprogramming OR "macro-programming” OR "declarative approach" OR "imperative approach" OR "programming abstraction") AND ("high level") AND ("internet of things" OR "sensor networks")

Para minimizar vieses na definição da string de busca, pesquisadores em IoT e em MSL auxiliaram esta pesquisa. Também na seleção dos artigos, houve a presença do pesquisador em IoT como árbitro principal, cuja atuação foi fundamental nos casos em que houve dificuldade de decidir pela seleção dos estudos primários.

Por conseguinte, foram definidos critérios de inclusão (CI) e exclusão (CE) para os estudos encontrados na atividade de busca. O único CI determina que, após a leitura dos metadados de cada estudo, este será incluído caso reporte o uso de abstração na programação e de adaptação na infraestrutura em domínios de aplicação de IoT e WSN. Oito CEs foram elaborados de modo que para que um estudo seja eliminado, basta que ele se encaixe em um CE. Dentre os CEs definidos, incluem-se a aplicação de filtro temporal às buscas, entre 2004 e 2020, o idioma obrigatoriamente na língua inglesa, dentre outros.

Já na fase de condução do MSL, foram retornados 111 trabalhos após a aplicação da string de busca ${ }^{3}$ nas fontes citadas: 16 da ACM Digital Library, 15 da IEEE Xplore e 80 da Scopus. Destes, 27 tratavam-se de estudos duplicados, totalizando 84 publicações para avaliação. Após a leitura dos metadados desses 84 estudos e a aplicação simultânea dos $\mathrm{CI}$ e CE, chegou-se a 38 artigos para leitura na íntegra. A Figura 1 ilustra a quantidade de estudos identificados, duplicados e selecionados neste MSL. Detalhes do protocolo sobre os estudos eliminados e a identificação de estudos duplicados também estão disponíveis ${ }^{4}$.

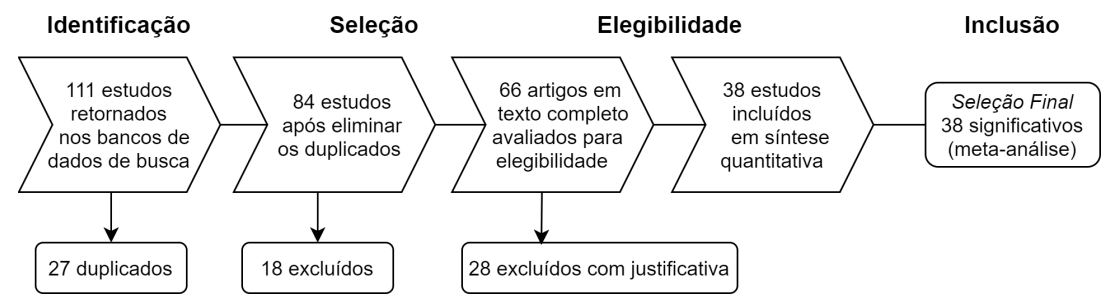

Figura 1. Fluxo de informação com as etapas e quantitativo de estudos aceitos.

Esses 38 artigos foram então submetidos à leitura de seus textos completos. De forma similar, o CI e os CE foram novamente aplicados para uma eventual equívoco de estudo incluído pela leitura preliminar de seus metadados. Nenhum dos 38 estudos foi eliminado nesta etapa.

Com essa leitura na íntegra, foram extraídas informações consideradas relevantes de cada pesquisa, a saber: área de conhecimento, domínio de aplicação, ano e veículo de publicação, nível de adaptação e tipo de abstração utilizados, detalhes sobre validação experimental, limitações e trabalhos futuros. A escolha dessas informações foi devidamente planejada como item do protocolo definido na primeira etapa deste MSL.

A lista contendo os 38 estudos relevantes para esta pesquisa encontra-se acessível $^{5}$. Trinta e sete foram encontrados na base Scopus, havendo estudos duplicados nas bases da ACM e do IEEE. Portanto, para o contexto deste MSL, a base Scopus mostrou-se a mais representativa.

\footnotetext{
${ }^{3}$ A busca em todas as bibliotecas digitais deu-se em 14/07/2020.

${ }^{4}$ Disponível para acesso em: http: //bit. ly/3cTsHKo

${ }^{5}$ Disponível para acesso em: http://bit.1y/3qCNKWq
} 


\section{Resultados e Discussão}

Esta seção apresenta a análise e a síntese das informações extraídas de cada um dos 38 estudos selecionados. O resultado das atividades de análise e síntese permite responder as QPs deste MSL, como apresentado a seguir.

QP1: Quanto à macroprogramação, quais áreas de aplicação são enfocadas?

Verificou-se que $73,7 \%$ dos trabalhos (28 de 38) abordam exclusivamente WSN e o restante trata macroprogramação em ambos ou especificamente em IoT (ambos com 13,2\%). Estudos exclusivos de macroprogramação em IoT iniciaram a partir de 2015 e, em 2019, todos focam em IoT. Essa tendência de crescimento por macroprogramação em IoT é corroborado por [Greer et al. 2019]. Dado que a busca por estudos deu-se em meados de 2020, é possível que mais estudos nesse tema tenham sido publicados.

Quanto ao domínio de aplicação, 90\% dos trabalhos (34 de 38) possuíam propósito geral de aplicação, percentual aproximado dos artigos que realizaram estudos de casos reais (31). Destes, concluiu-se que 13 campos de aplicações foram abordados, como mostra a Figura 2. Há trabalhos que não efetuaram aplicação desta forma, logo não foi possível sua catalogação (p.ex. artigos de 2004 e 2015), enquanto outros não o fizeram em um campo específico, a exemplo de estudos de caso usando hardware e software específicos sem referenciar um campo onde a proposta foi verificada experimentalmente (representados por "outros").

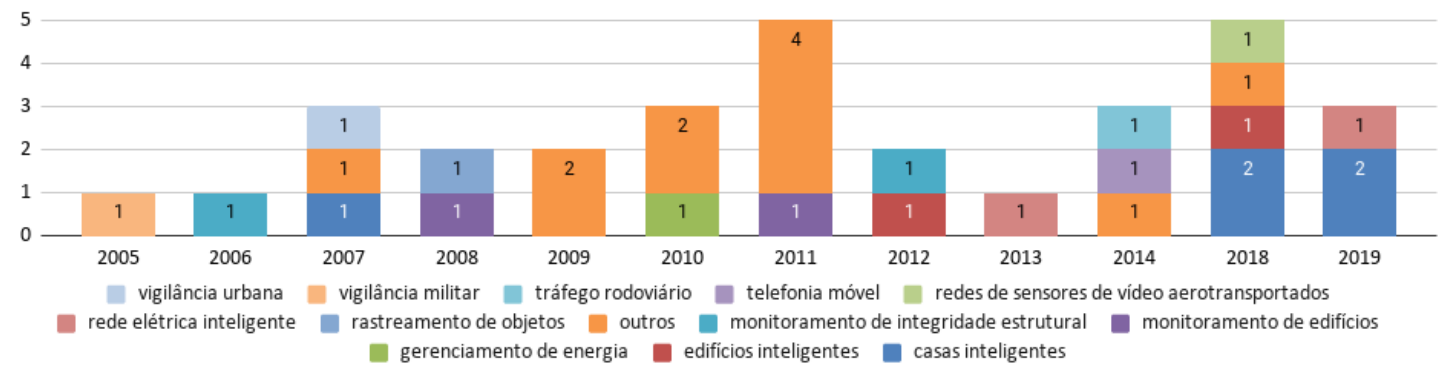

Figura 2. Estudo de caso por ano de publicação.

QP2: Quando e em quais veículos os estudos primários têm sido publicados?

Os anos com maior índice de publicações aceitas foram 2011 e 2018, enquanto que nenhum estudo foi retornado em 2016, 2017 e 2020. Verificou-se também elevada diversidade das conferências, workshops e journals, sendo 36 delas distintas. Os únicos veículos que tiveram duas publicações cada foram a ACM/IEEE International Conference on Information Processing in Sensor Networks (IPSN) e a IEEE Conference on Local Computer Networks Workshops (IEEE LCN).

Percebe-se, com estes resultados, que não há ainda uma comunidade consolidada no assunto, convergindo para eventos específicos, ao passo que uma grande pluralidade de conferências tem explorado a temática de macroprogramação, o que também pode ser verificado temporalmente por tipos de veículos de publicação heterogêneos.

QP3: Em qual(is) nível(is) ocorrem a adaptação e qual(is) são os tipos de abstrações na infraestrutura? 
Esta QP visa avaliar o nível de adaptação e o tipo de abstração empregado. Seguiu-se a taxonomia de [Krupitzer et al. 2015], que categoriza o nível de adaptação em: aplicação (individual ou conjunto de aplicações), sistemas de software (middleware ou sistema operacional), comunicação (infraestrutura de rede ou padrões de comunicação), contexto e recursos técnicos.

Dentre esses níveis, o mais citado, com 12 artigos, referiu-se à comunicação na subdivisão de infraestrutura de rede $(31,6 \%)$, seguida de adaptação sensível ao contexto $(23,7 \%)$, com 9 estudos. De forma geral, comunicação foi o nível com maior adaptação encontrado nos trabalhos. Além disso, considerando a distribuição temporal dos estudos relevantes, verificou-se a predominância de infraestrutura de rede entre os anos de 2009 a 2014; contudo, este item não foi catalogado mais recentemente (2019).

Ainda quanto ao nível de adaptação, este foi analisado juntamente de área de conhecimento (Figura 3). Entre os artigos, verificando-se especialmente a diferenciação entre IoT e WSN, o primeiro possuiu, em maioria, adaptações no nível de aplicação, considerando um conjunto de aplicações (2 estudos) e o segundo, no nível de comunicação, quanto à infraestrutura de rede (11 trabalhos). Não foram encontrados artigos que realizassem a adaptação no nível de recursos técnicos, que refere-se à hardware e similares.

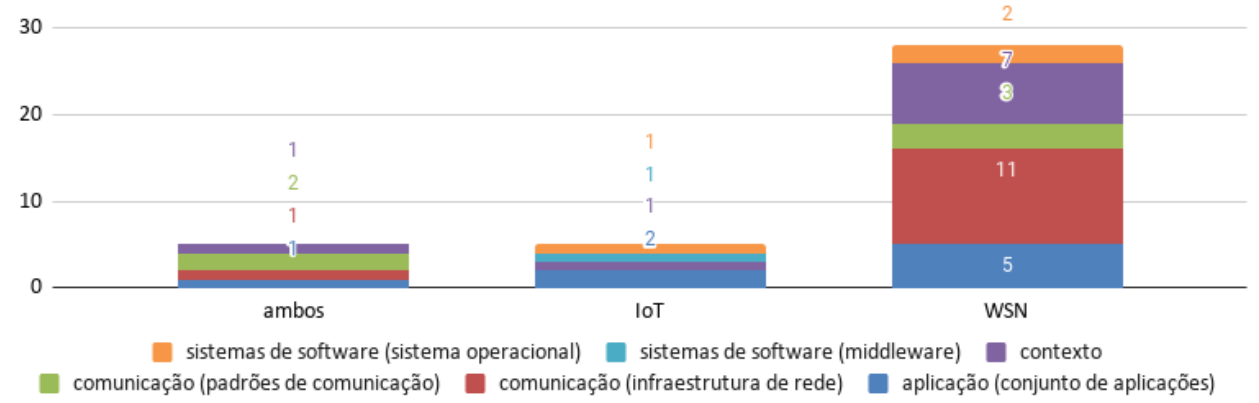

Figura 3. Nível de adaptação por área de conhecimento.

Quanto aos tipos de abstração, adotou-se a abordagem de [Mottola 2008], que os classifica em nó, grupo, ou sistema. O nó representa construções que alteram o estado de nós individuais; no grupo, altera-se um subconjunto de nós; quando centrado no sistema, os efeitos de uma instrução são capazes de perpetuar por toda a rede.

Observou-se que o tipo "grupo", abarcou 55\% das publicações (21 artigos). Ademais, ao verificar por ano, percebeu-se que esta classificação esteve presente em 11 dos 14 anos analisados. Acredita-se que esse quantitativo de aplicações centradas no grupo seja decorrente da maior flexibilidade provida ao subdividir, por exemplo, uma rede de sensores, com elementos dispostos em grupos menores com características comuns.

Por fim, quando averiguado o nível de adaptação e o tipo de abstração de maneira conjunta, destaca-se que tanto para o grupo quanto para o nó, a infraestrutura de rede foi o nível de adaptação mais empregado. Já considerando o sistema, adaptações quanto ao contexto foram mais relevantes, como apresentado na Figura 4.

QP4: Como adaptações são realizadas em pesquisas aplicadas a WSN e IoT?

Avaliando a proposta desempenhada nos artigos, 12 itens distintos foram elencados e framework representou 34\% dos itens citados (13 trabalhos), sendo a opção mais 


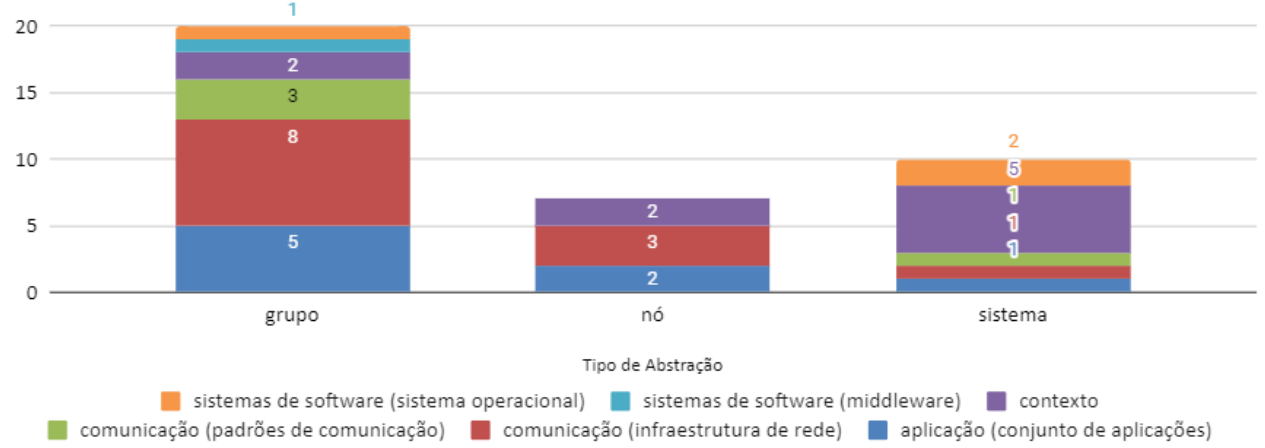

Figura 4. Nível de adaptação por classificação da abstração.

recorrente. Dado o intuito geral de um framework de facilitar o desenvolvimento de aplicações, este se correlaciona diretamente à macroprogramação.

Contudo, ao averiguar de forma temporal, apesar de um crescimento parcialmente linear de 2007 a 2011, a partir deste último ano, parou de figurar como uma parcela significativa das propostas empregadas. Consecutivamente, linguagens de programação foram o segundo tipo de proposta mais abordado depois de frameworks.

Ademais, em prol de compreender o rigor científico das distintas propostas levantadas, também foi verificado a forma empregada por cada um dos trabalhos para validação experimental. Dividindo-se em implementação, testbed, simulação e protótipo, em suma, implementação recebeu o percentual de 63,2\% dentre os analisados (24 artigos). Destacase forte presença deste formato pelo fato dos trabalhos tratarem de programação com adaptação em abstrações de alto nível (macroprogramação), sendo a implementação uma forma usual de evidenciar este uso. Por ano, percebeu-se que validações do tipo testbed foram pouco exploradas (intervalo de tempo esparso entre suas utilizações).

Também efetuaram-se análises da validação das propostas desses estudos. Foi possível verificar os meios de validação mais utilizados nas distintas propostas. A exemplo disso, para biblioteca, framework, interface, linguagem, máquina virtual, middleware e sistema, a implementação destacou-se entre os tipos aplicados. Outro ponto refere-se quanto ao uso de testbed, o qual somente foi visualizado em propostas de frameworks.

QP5: Quais são os problemas apontados no contexto da adaptabilidade nos domínios de aplicação estudados?

Buscou-se verificar a presença dos principais problemas apontados no contexto de adaptação em decorrência das áreas de aplicação. Entretanto, verificando-se as limitações descritas pelos autores(as) nas publicações, 21 itens distintos foram citados, e aspectos de comunicação figuraram com maior quantitativo, representando um percentual de 15,4\%. Em maior número, as limitações relacionadas a problemas de comunicação foram obtidas dentro da área de IoT (4 trabalhos), enquanto para WSN referem-se a middleware e estudos que ainda estão em desenvolvimento ( 2 trabalhos cada).

Acerca dos trabalhos futuros, os mais citados foram: tradutor de código, framework, aplicação em ambientes maiores e mudanças em tempo de execução. Logo, verifica-se uma tendência por trabalhos que envolvam abstrações explorando o sistema como um todo e inclusive, levando em conta adaptações de contexto, as quais apareceram 
Tabela 1. Realizações futuras por publicações e ano.

\begin{tabular}{|c|c|c|c|c|c|}
\hline Trabalho futuro & Ano(s) & Artigo(s) & Trabalho futuro & Ano(s) & Artigo(s) \\
\hline minimização de restrições & 2004 & S32 & localização dinâmica & 2005 & S11 \\
\hline liberação para domínio público & 2006 & S33 & modelo analítico para roteamento & 2006 & S30 \\
\hline balanceamento de carga & 2007 & S19 & qualidade dos requisitos & 2007 & S6 \\
\hline mapeamento de ambientes urbanos & 2007 & S8 & manutenção da estrutura & 2007 & S29 \\
\hline tradutor de código & $2008 / 2009$ & S37, S5 & execução realista & 2009 & S22 \\
\hline propagação de programas & 2009 & S34 & menor complexidade de heurísticas & 2010 & S20 \\
\hline ambientes maiores & $2010 / 2015$ & $\mathrm{~S} 27, \mathrm{~S} 3$ & comunicação remota & 2010 & S1 \\
\hline integração com protocolos & 2011 & S4 & implementação com XML & 2011 & S21 \\
\hline política de substituição de código & 2011 & S17 & ambiente de tempo de execução & 2011 & S38 \\
\hline eliminar redundância de tarefas & 2011 & S23 & modelagem 3D & 2012 & S10 \\
\hline integração de linguagens & 2012 & S36 & avaliação de robustez & 2014 & S9 \\
\hline adequação à restrição de recursos & 2014 & S15 & framework & $2014 / 2018$ & S3, S13 \\
\hline reprogramação sem fio & 2014 & S26 & tempo de execução & $2018 / 2019$ & $\mathrm{~S} 12, \mathrm{~S} 14$ \\
\hline aplicativos inteligentes & 2019 & S28 & interação de dispositivos & 2019 & S35 \\
\hline implantação de demonstradores & 2019 & S25 & & & \\
\hline
\end{tabular}

como indícios nos estudos mais recentes. A Tabela 1 exibe as principais atividades como desafios futuros, considerando a variedade de temáticas, com identificação segundo a lista de trabalhos aceitos.

Como resultado final deste MSL, a Figura 5 descreve um mapa que correlaciona o nível de adaptação, a área de conhecimento e o tipo de abstração utilizado. Ao analisar-se pelo quantitativo de publicações, fica evidente a expressiva adaptação quanto à comunicação no âmbito de infraestrutura de rede para WSN com abstração no grupo. Quando considerado o caso específico de IoT, foram elencadas, em maior número, adaptações no nível de aplicação, a qual é vista comumente como uma das camadas mais altas de abstração, e uma possível tendência para a área.

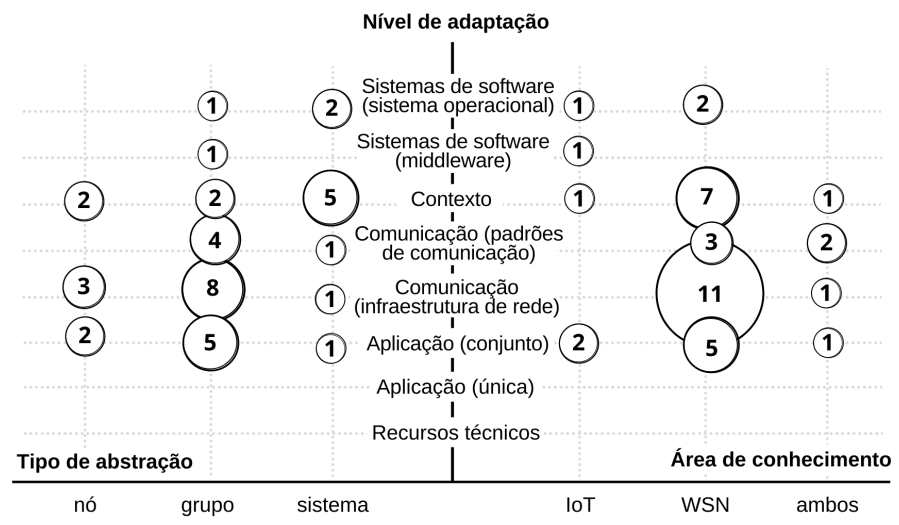

Figura 5. Mapeamento: nível de adaptação por tipo de abstração e área.

\section{Considerações Finais}

Os maiores benefícios obtidos com a realização de um MSL é fornecer uma visão geral da literatura sobre um tema, descobrir oportunidades de pesquisa, aprender com os estudos encontrados e auxiliar na definição de temas de pesquisas de mestrado ou doutorado.

Como uma tendência para pesquisadores e programadores, foi possível observar que a macroprogramação apresenta uma abstração simples e passível para não especialistas, sem a necessidade de compreensão explícita da tecnologia envolvida que constrói o sistema. A disponibilidade de abstrações para apresentar soluções para essa semântica 
e de experiência pode ser particularmente desejável em configurações de IoT porque as implantações tendem a envolver uma ampla gama de partes interessadas de diferentes origens e interagir em um relacionamento muito próximo com os usuários finais e seus ambientes. Entretanto, os resultados deste estudo sinalizam, que ainda é uma questão em aberto, relativamente poucas abordagens de macroprogramação são projetadas para preencher essa lacuna, com a maioria voltada para desenvolvedores de sistemas.

Salienta-se que apesar da área de WSN possuir maior número de publicações, IoT vem aparecendo nos estudos recentes de modo mais expressivo [Chen et al. 2019, D'Urso et al. 2019, Sengupta et al. 2019], como proeminente campo de pesquisa (QP1).

Dentre os anos analisados, 2011 e 2018 situaram-se com maior número de estudos, ao passo que não foi verificada congruência entre os veículos de publicação, o que evidencia a pluralidade de veículos para a disseminação de trabalhos neste cenário (QP2).

Foi também identificada forte presença de adaptação em infraestrutura de rede com abstração centrada no grupo (QP3), além de um grande número de propostas de frameworks adotadas com implementação prática (QP4), a exemplo de [Pathak and Prasanna 2010, Mamei 2011, Gupta et al. 2011].

Acerca das limitações elencadas, a maioria referiu-se a problemas de comunicação, enquanto trabalhos futuros incluem adaptações em tempo de execução (QP5). É possível identificar que nos trabalhos atuais a macroprogramação vem sendo proposta como adaptação em tempo de execução [Mizzi et al. 2018, Baghli et al. 2018], demonstrando uma tendência de pesquisa passível de exploração.

Por fim, como trabalhos futuros objetiva-se atualizar este MSL com uma busca mais ampla, combinando-se estratégias de coleta manual por veículos que apresentaram mais artigos, além de prover mais pesquisas relacionadas, a exemplo de uma revisão sistemática com enfoque em sistemas adaptativos ao contexto.

\section{Referências}

Awan, A., Jagannathan, S., and Grama, A. (2007). Macroprogramming Heterogeneous Sensor Networks using COSMOS. Operating Systems Review (ACM), pages 159-172.

Baghli, R. B., Najm, E., and Traverson, B. (2018). Defining Services and Service Orchestrators Acting on Shared Sensors and Actuators. MODELSWARD 2018 - Proceedings of the 6th International Conference on Model-Driven Engineering and Software Development, 2018-Janua(Modelsward):237-246.

Chen, J., Cañete, E., Garrido, D., Díaz, M., and Piotrowski, K. (2019). PICO: A platform independent communications middleware for heterogeneous devices in smart grids. Computer Standards and Interfaces, 65(February 2018):1-14.

Choochaisri, S., Pornprasitsakul, N., and Intanagonwiwat, C. (2012). Logic macroprogramming for wireless sensor networks. International Journal of Distributed Sensor Networks, 2012.

D’Urso, F., Longo, C. F., and Santoro, C. (2019). Programming Intelligent IoT Systems with a Python-based Declarative Tool. CEUR Workshop Proceedings, 2502(November). 
Greer, C., Burns, M., Wollman, D., and Griffor, E. (2019). Cyber Physical Systems and Internet of Things in Industry. NIST Special Publication, pages 2839-2840.

Gupta, V., Kim, J., Pandya, A., Lakshmanan, K., Rajkumar, R., and Tovar, E. (2011). Nano-CF: A coordination framework for macro-programming in Wireless Sensor Networks. 2011 8th Annual IEEE Communications Society Conference on Sensor, Mesh and Ad Hoc Communications and Networks, SECON 2011, pages 467-475.

Hussein, M., Li, S., and Radermacher, A. (2017). Model-driven development of adaptive IoT systems. CEUR Workshop Proceedings, 2019:17-23.

Krupitzer, C., Breitbach, M., Roth, F. M., VanSyckel, S., Schiele, G., and Becker, C. (2015). A survey on engineering approaches for self-adaptive systems. Pervasive and Mobile Computing Journal, 17:184-206.

Lepekhin, A., Borremans, A., Ilin, I., and Jantunen, S. (2019). A systematic mapping study on internet of things challenges. International Workshop on Software Engineering Research and Practices for the Internet of Things, pages 9-16.

Mamei, M. (2011). Macro programming a spatial computer with bayesian networks. ACM Transactions on Autonomous and Adaptive Systems, 6(2):1-25.

Mizzi, A., Ellul, J., and Pace, G. (2018). D’Artagnan: An Embedded DSL Framework for Distributed Embedded Systems. Proceedings of the Real World Domain Specific Languages Workshop 2018, pages 1-9.

Mottola, L. (2008). Programming wireless sensor networks: from physical to logical neighborhoods. Thesys, Politecnico di Milano (Italy).

Mottola, L. and Picco, G. P. (2011). Programming wireless sensor networks: Fundamental concepts and state of the art. ACM Computing Surveys, 43(3).

Newton, R. and Welsh, M. (2004). Region streams: Functional macroprogramming for sensor networks. ACM International Conference Proceeding Series, 72:78-87.

Pathak, A. and Prasanna, V. K. (2010). Energy-efficient task mapping for data-driven sensor network macroprogramming. IEEE Transactions on Computers, 59(7):955968.

Petersen, K., Vakkalanka, S., and Kuzniarz, L. (2015). Guidelines for conducting systematic mapping studies in software engineering: An update. Information and Software Technology, 64:1-18.

Sengupta, A., Leesatapornwongsa, T., Ardekani, M. S., and Stuardo, C. A. (2019). Transactuations: Where transactions meet the physical world. Proceedings of the 2019 USENIX Annual Technical Conference, USENIX ATC 2019, pages 91-105.

Sethi, P. and Sarangi, S. R. (2017). Internet of Things: Architectures, Protocols, and Applications. Journal of Electrical and Computer Engineering, (January).

Sugihara, R. and Gupta, R. K. (2008). Programming models for sensor networks: A survey. ACM Transactions on Sensor Networks, 4(2):1-27. 